\title{
Modeling of a high-power thruster with anode layer
}

\author{
Michael Keidara) and lain D. Boyd \\ Department of Aerospace Engineering, University of Michigan, Ann Arbor, Michigan 48109
}

Isak I. Beilis

Electrical Discharge and Plasma Laboratory, Fleischaman Faculty of Engineering, Interdisciplinary Department, Tel Aviv University, PO Box 39040, Tel Aviv 69978, Israel

(Received 29 October 2003; accepted 15 January 2004; published online 5 April 2004)

\begin{abstract}
Among Hall thruster technologies, the thruster with anode layer (TAL) has much wider technical capabilities, especially in the high-power regime of operation. In this paper, various aspects of the plasma flow in a high-power thruster with anode layer are studied. Based on a 2D hydrodynamic model, the formation of a space-charge sheath near the acceleration channel wall and the sheath expansion in the acceleration channel are calculated. It is found that the high-voltage sheath near the channel wall expands significantly and the quasineutral plasma region is confined in the middle of the channel. For instance, in the case of a $3 \mathrm{kV}$ discharge voltage, the sheath thickness is about $1 \mathrm{~cm}$, which is a significant portion of the channel width (which is typically a few $\mathrm{cm}$ ). In addition, a simplified quasi-1D model is developed to study the anode acceleration layer, which is confined by channel walls. It is found that near-wall sheath expansion leads to an increase in current density along the channel, and this in turn causes decrease of the acceleration region length. This is an important finding as it has implications for high-power TAL behavior, in which contact of the plasma with acceleration channel walls can be limited. (C) 2004 American Institute of Physics.
\end{abstract}

[DOI: $10.1063 / 1.1668642]$

\section{INTRODUCTION}

Hall thrusters are among the most advanced and efficient types of electrostatic propulsion devices. The Hall thruster configuration is beneficial because the particle acceleration takes place in a quasineutral plasma and thus is not limited by space-charge effects. The electrical discharge in the Hall thruster occurs across the external magnetic field, which has a predominantly radial component, and ions are accelerated along the axial electric field. Passing the electron current across a magnetic field leads to an electron closed drift or Hall drift, that provides the necessary gas ionization. The original idea of ion acceleration in the quasineutral plasma was introduced in the mid 1960s (see Refs. 1-4), and since then numerous experimental and theoretical investigations have been conducted. The main results of these studies were summarized in recent reviews. ${ }^{5,6}$ Generally, two different types of Hall thruster were developed: a thruster with closed electron drift and extended acceleration zone, or stationary plasma thruster (SPT), and a thruster with short acceleration channel or thruster with anode layer (TAL) ${ }^{4,5}$ In an SPT, the interaction of the plasma with the dielectric wall plays an important role. Due to the collisions of the electrons with the wall and secondary electron emission, the electron temperature remains relatively low in comparison to the TAL. As a result, the ion acceleration occurs over a more extended region. ${ }^{5,7}$ Besides, experimental observations show that the various dielectric materials affect the discharge behavior in the Hall thruster. ${ }^{8}$ In addition, Raitses et al..$^{9,10}$ found that use of sectioned metal electrodes inside the Hall thruster channel



has a substantial effect on the discharge characteristics as well as on thruster performance. Therefore, it is natural that having acceleration channel walls made from conductive material would significantly affect Hall thruster operation. In fact, in a TAL, the ion acceleration takes place over a very short length of about the electron Larmor radius near the anode. ${ }^{5}$ This is why the term "anode layer" was attributed to the Hall thruster with metal walls. Similarly, in magnetically insulated ion diodes, ion acceleration occurs in a thin layer having thickness of about the electron Larmor radius. ${ }^{11}$ Despite many theoretical efforts, the complicated physical processes in the Hall thruster channel are far from being completely understood. Mainly, the physics of the electron transport, plasma interaction with the wall, and the transition between the quasineutral plasma and the sheath have not been investigated in satisfactory detail.

In the TAL variant, an electric discharge in the crossed magnetic and electric fields is created in the gap between magnetic poles where closed electron drift takes place. In this gap, the neutral atoms are ionized and accelerated. Some fraction of the accelerated ion stream is directed toward the wall that limits the discharge in the radial direction and protects the magnetic poles from erosion. This leads to wear of these walls due to ion bombardment and results in a shortened lifetime of such accelerators.

Recently, great emphasis was put forward into development of high-power Hall thrusters. ${ }^{12}$ Among Hall thruster technologies, the TAL configuration seems to have much wider technical capabilities and range of parameters. ${ }^{13}$ Since reduced erosion of various thruster components, such as electrodes, insulators, screens, etc., is critical for the long- 


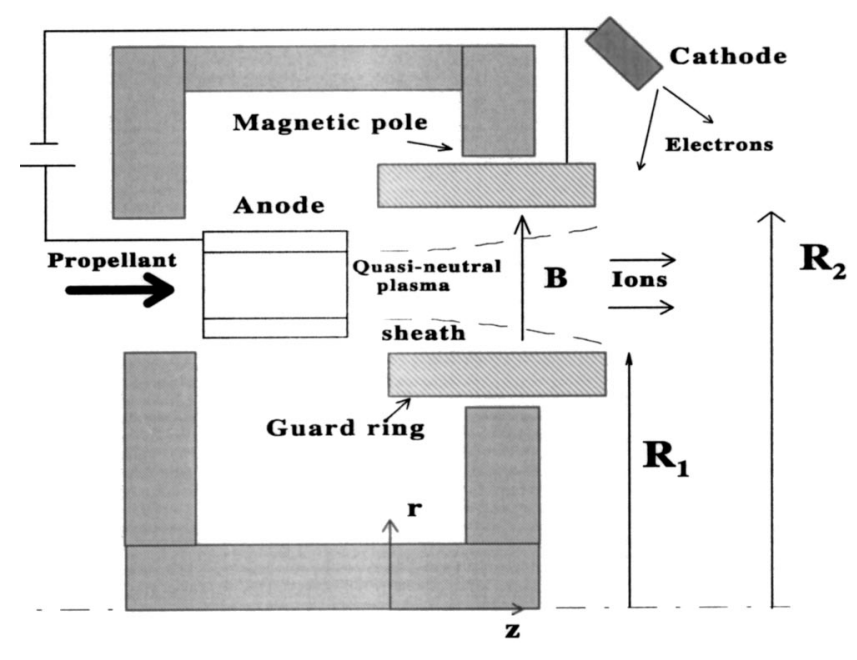

FIG. 1. Schematic of the acceleration channel in a TAL.

term operation of the thruster, the TAL variant of Hall thruster technology seems to be beneficial since it has a very small acceleration region and therefore small area with contact of ions with materials where possible erosion occurs. In order to meet high-power requirements, a thruster with anode layer (TAL) was developed and an experimental model was presented. ${ }^{13}$ TAL using bismuth as a propellant demonstrated specific impulse in the range of 2000-5000 s at power levels of $10-34 \mathrm{~kW}$.

The idealized picture of the Hall thruster in which ions are accelerated in the channel is not exact and, in reality, in TALs as well as in some SPTs significant ion acceleration occurs outside of the channel in the fringing magnetic field. This may be considered as one possible mechanism of high plasma beam divergence in Hall thrusters. ${ }^{14}$

TALs can operate in both single and two-stage regimes; in the two-stage regime, ionization and acceleration take place in two separate discharges, so that at the exit plane of the first stage the plasma is highly ionized and additional ionization in the second stage is insignificant. ${ }^{13}$ Usually, the two-stage regime of operation has many advantages, for instance the possibility to achieve much higher specific impulse. ${ }^{15}$ When a two-stage thruster is considered, the most important region in terms of long-time operation is the second acceleration stage, in which a very large voltage drop is applied. The second stage channel geometry is shown schematically in Fig. 1.

An important aspect of high-power TAL is the sheath formation near the channel wall. Since the channel walls have potential, which is equal to the cathode potential, a significant potential drop can exist between the wall and the plasma. As a result, a high-voltage space-charge sheath is formed and the sheath thickness can be comparable to the channel width. Therefore, a transition between quasineutral and space-charge regimes can occur in the high-power TAL channel. This transition can have a significant effect on the TAL operation and is therefore extremely important for practical applications. In this paper this effect will be considered in detail.

In this paper we describe plasma flow in the second stage of the TAL. The paper is organized as follows. The plasma-wall transition approach is described in Sec. II. In Sec. III a hydrodynamic model of the quasineutral plasma is developed. A specific example of high-power TAL is described in Sec. IV. In addition, a simplified quasi-1D model of the anode layer that includes plasma-wall transition phenomena is developed in Sec. V.

\section{ON THE PLASMA-WALL TRANSITION}

The plasma-wall transition region in the TAL channel determines the particle and energy fluxes from the plasma to the wall. Recently, we presented a model of plasma wall transition in the SPT that accounts for secondary electron emission. ${ }^{16}$ In order to develop a self-consistent model, the boundary parameters at the sheath edge (ion velocity and electric field) are obtained from a two-dimensional plasma bulk model. In the considered condition, i.e., ion temperature much smaller than that of electrons and significant ion acceleration in the axial direction, the presheath scale length becomes comparable to the channel width so that the plasma channel becomes an effective presheath. It was shown that a plasma-sheath matching approach proposed previously could be used. ${ }^{17}$ In this approach, the electric field that develops in the presheath can serve as a boundary condition for the sheath. At the same time the model predicts that the quasineutrality assumption at the presheath edge is still valid in typical cases.

It was shown ${ }^{16}$ that the presheath scale length becomes comparable to the channel width under typical conditions of the Hall thruster channel. Thus, the model for the quasineutral plasma region is extended up to the sheath edge in order to provide the boundary condition at the plasma-sheath interface.

The density $N_{s}$ and velocity $V_{s}$ at the presheath-sheath interface serve as a boundary condition for the quasineutral plasma model that is described in the next section.

\section{HYDRODYNAMIC MODEL OF THE QUASINEUTRAL PLASMA FLOW}

The model is based on the assumption that the quasineutral region length (i.e., channel width, see Fig. 1) is much larger than the Debye radius, and therefore we will assume that $Z_{i} n_{i}=n_{e}=n$, where $Z_{i}$ is the ion mean charge, $n_{i}$ is the ion density, and $n_{e}$ is the electron density. For simplicity only singly charged ions are considered in this paper $\left(Z_{i}=1\right)$. We will consider the plasma flow in an annular channel as shown in Fig. 1. A magnetic field with only a radial component, $B_{r}=B$, is imposed. Cylindrical coordinates will be used with angle $\theta$, radius $r$, and axial distance from the anode $z$, respectively. The plasma presheath-sheath interface is considered to be the lateral boundary for the plasma flow region. A plasma will be considered with "magnetized" electrons and "unmagnetized" ions, i.e., $\rho_{e} \ll L \ll \rho_{i}$, where $\rho_{e}$ and $\rho_{i}$ are the Larmor radii for the electrons and ions, respectively, and $L$ is the channel length. We employ a hydrodynamic model assuming: (i) the system reaches a steady state, and (ii) the electron component is not inertial, i.e., $\left(\mathrm{V}_{\mathrm{e}} \nabla\right) \cdot \mathrm{V}_{\mathrm{e}}=0$. 
Generally, two regimes are possible in a TAL, the socalled vacuum regime (in which the electron density is much higher than that of ions) and the quasineutral plasma regime. ${ }^{18}$ Below, we briefly formulate a model for the quasineutral regime. A hydrodynamic model is employed in a 2D domain assuming that the system reaches a steady state. The momentum and mass conservation equations for electrons, ions, and neutrals under these conditions have the following form:

$$
\begin{aligned}
& n m_{i}\left(V_{i} \nabla\right) V_{i}=n e E-\nabla P_{i}-\beta n m_{i} n_{a}\left(V_{i}-V_{a}\right), \\
& 0=-e n\left(E+V_{e} \times B\right)-\nabla P_{e}-n \nu_{\mathrm{ef}} m_{e} V_{e}, \\
& \nabla \cdot\left(V_{i} n\right)=\beta n n_{a}, \\
& \nabla \cdot\left(V_{a} n_{a}\right)=-\beta n n_{a}, \\
& \frac{3}{2} \partial\left(j_{e} T_{e}\right) / \partial z=Q_{j}-Q_{w}-Q_{\text {ion }},
\end{aligned}
$$

where $i, a, e$ are subscripts for ions, neutral atoms, and electrons, respectively, $\beta$ is the ionization rate, $\nu_{\text {ef }}$ is the effective electron collision frequency, $V$ is the velocity, $Q_{j}=j_{e} E$ is the Joule heat, $E$ is the axial component of the electric field, $j_{e}$ is the electron current density, $Q_{w}=\nu_{w} n\left(2 e T_{e}+e \Delta \varphi_{w}\right)$ represents the energy plasma losses to the walls, ${ }^{16} \Delta \varphi_{w}$ is the voltage drop across the sheath near the channel wall, $\nu_{w}$ is the frequency of electron collisions with walls, $Q_{\text {ion }}$ $=e n_{a} n U_{i} \beta$ represents ionization energy losses, and $U_{i}$ is the ionization potential. To simplify the problem without missing the major physical effects, we consider one-dimensional flow of the neutrals, i.e., $V_{a}=V_{a z}$. The equations for the heavy particles (ions and neutrals) may be written in component form in cylindrical coordinates by noting that the ion temperature is much smaller than the electron temperature (which makes it possible to neglect the ion pressure term in the momentum conservation equation)

$$
\begin{aligned}
& \frac{\partial\left(n V_{z}\right)}{\partial z}+\frac{\partial\left(n V_{r}\right)}{\partial r}+\frac{n V_{r}}{r}=\beta n n_{a}, \\
& V_{z} \frac{\partial V_{z}}{\partial z}=-V_{r} \frac{\partial V_{z}}{\partial r}+\frac{e}{m_{i}} E_{z}-\beta\left(V_{z}-V_{a}\right) n_{a}, \\
& V_{z} \frac{\partial V_{r}}{\partial z}=-V_{r} \frac{\partial V_{r}}{\partial r}+\frac{e}{m_{i}} E_{r}, \\
& \frac{\partial\left(n_{a} V_{a}\right)}{\partial z}=-\beta n n_{a} .
\end{aligned}
$$

In the system of equations (6)-(9) the subscript for ions was omitted, i.e., $V=V_{i}$. In this model the electron flow [Eq. (2)] will be considered separately along and across magnetic field lines. Due to the configuration of the magnetic field (i.e., only the radial magnetic field component is considered in the model), the electron transport is greater in the azimuthal direction $(E \times B$ drift) than in the axial direction (drift diffusion due to collisions). According to Eq. (2), the electron transport equation along the magnetic field can be written as a balance between pressure and electric forces assuming that the current component in the radial direction is small (i.e., $j / \sigma \ll E$, cold quasineutral beam approximation ${ }^{19}$ ). If we assume that the electron temperature is constant along each magnetic field line, we obtain that

$$
\varphi-T_{e} \ln n=\text { const. }
$$

The left-hand side of this equation is known as a thermalized potential. ${ }^{6}$ This equation makes it possible to reduce the twodimensional calculation of the electric field to a onedimensional problem. According to Eq. (10), the electric field in the radial direction $E_{r}$ is determined by the electron pressure gradient in this direction. Calculating the potential distribution along the channel centerline makes it possible to calculate the potential in the entire domain using Eq. (10), similar to Ref. 16. For known total discharge current and ion current fraction one can calculate the electron current fraction from the current continuity condition. The equation describing the electron transport across the magnetic field can be obtained from Eq. (2), and reads

$$
j_{e z}=e n \frac{\frac{e}{m \nu_{e f}}}{1+\left(\frac{\omega_{e}}{\nu_{\mathrm{ef}}}\right)^{2}}\left(E_{z}+\frac{\partial T_{e}}{\partial z}+T_{e} \frac{\partial \ln n}{\partial z}\right),
$$

where $\nu_{\text {ef }}=\nu_{e n}+\nu_{e w}+\nu_{B}$ is the effective electron collision frequency. The different electron collision mechanisms are described below.

\section{A. Electron collisions}

For typical conditions of the Hall thruster, the effect of Coulomb collisions appears to be negligibly small ${ }^{20}$ and will not be considered here. The total electron collision frequency considered in the present model consists of electron-neutral collisions and anomalous collisions (Bohm diffusion). The electron-neutral collision frequency may be estimated as follows:

$$
\nu_{e n}=n_{a} \sigma_{e a} V_{\mathrm{th}}^{e},
$$

where $n_{a}$ is the neutral density, $\sigma_{e a}$ is the collision cross section dependent on the electron energy, and $V_{t h}^{e}$ is the electron thermal velocity.

However, only including the classical mechanism of collisions cannot explain the electron transport observed in a Hall thruster. This was recognized long ago by many authors. ${ }^{5,6}$ Until now, however, there has been no consensus about which of the possible mechanisms of electron transport is most significant in the Hall thruster. While in the SPT, electron collisions with the walls may play the major role, ${ }^{4}$ in the TAL, which does not have significant secondary electron emission, anomalous transport ${ }^{21}$ may be important. In the present work we will include anomalous (Bohm) transport. The effective electron collision frequency related to the anomalous transport (Bohm diffusion) can be estimated as

$$
\nu_{B}=\alpha \omega_{e},
$$

where $\alpha \sim 1 / 16$ is the Bohm empirical parameter in the classical formulation. It will be shown below that the exact value of this parameter affects the potential drop across the channel. The best fit with the experimental data of the potential 
drop for a given discharge current corresponds to $\alpha \sim 1 / 100$ instead of the classical value $\sim 1 / 16$. It should be noted that the conclusion derived by different authors was that the best fit with experiment corresponds to $\alpha \sim 1 / 80$ to $1 / 100$ (Refs. 16, 22, and 23).

\section{B. Boundary conditions}

In order to obtain a solution of the system of equations (6) -(13), the following boundary conditions must be specified. At the upstream boundary $(z=0)$ we specify the ion density and velocity similarly to Refs. 16 and 24 assuming sonic ion velocity (determined by electron temperature) at the entrance $(z=0$, see Fig. 1) to the second stage. This upstream condition implies that we are considering only supersonic plasma flow assuming that the transition from subsonic to supersonic flow ${ }^{16,24}$ occurs at the entrance to the second stage. The atom velocity near the anode is assumed to be $V_{o a}=2 \times 10^{2} \mathrm{~m} / \mathrm{s}$ (Ref. 20). For given atom velocity the atom density is calculated from the mass flow rate. At the downstream boundary (thruster exit plane, $z=L$ ) we specify an electron temperature of $T_{e}=10 \mathrm{eV}$. The plasma density at the entrance to the second stage is determined by the ionization rate in the first stage. Since in this model only a second stage is considered, we will use the plasma density at the starting plane $(\mathrm{z}=0)$ as a parameter. It was found that our simulation results agree well with experiment (discharge current, acceleration efficiency) when the ratio between plasma density and neutral density at $z=0$ is about 0.02 . The results shown below correspond to this specific value of plasma density at the starting plane. Boundary conditions for ion velocity and density at the walls are determined by considering plasma-sheath transition as described in Sec. II and elsewhere. ${ }^{16}$ Here, we just note that a relation between the boundary ion velocity and the electric field can be found by considering the plasma-sheath interface. Having electric field, the plasma density gradient at the plasma-sheath interface is calculated and is used as a boundary condition for the density. Finally, the discharge voltage is set, i.e., $\varphi(z=0)$ $=\varphi_{a}$ and $\varphi(z=L)=0$.

\section{Numerical method}

The numerical analysis is similar to that developed previously. ${ }^{25}$ We use the implicit two-layer method to solve the system of equations (6)-(9). These equations are approximated by a two-layer six-point scheme. The electron temperature distribution is calculated by iteration initially assuming a trial temperature distribution that satisfies the boundary conditions.

\section{HIGH-POWER BISMUTH TAL}

In this section we describe the simulation of a particular TAL design that uses bismuth as the propellant. The bismuth thruster was originally developed in the 1960s in the former USSR. ${ }^{13,18}$ It was demonstrated that specific impulse in the range of 2000-5000 s at power levels of 10-34 kW can be achieved. This thruster has a two-stage configuration in order to separate the ion production and acceleration zones. In this paper we will consider only the second stage. This requires
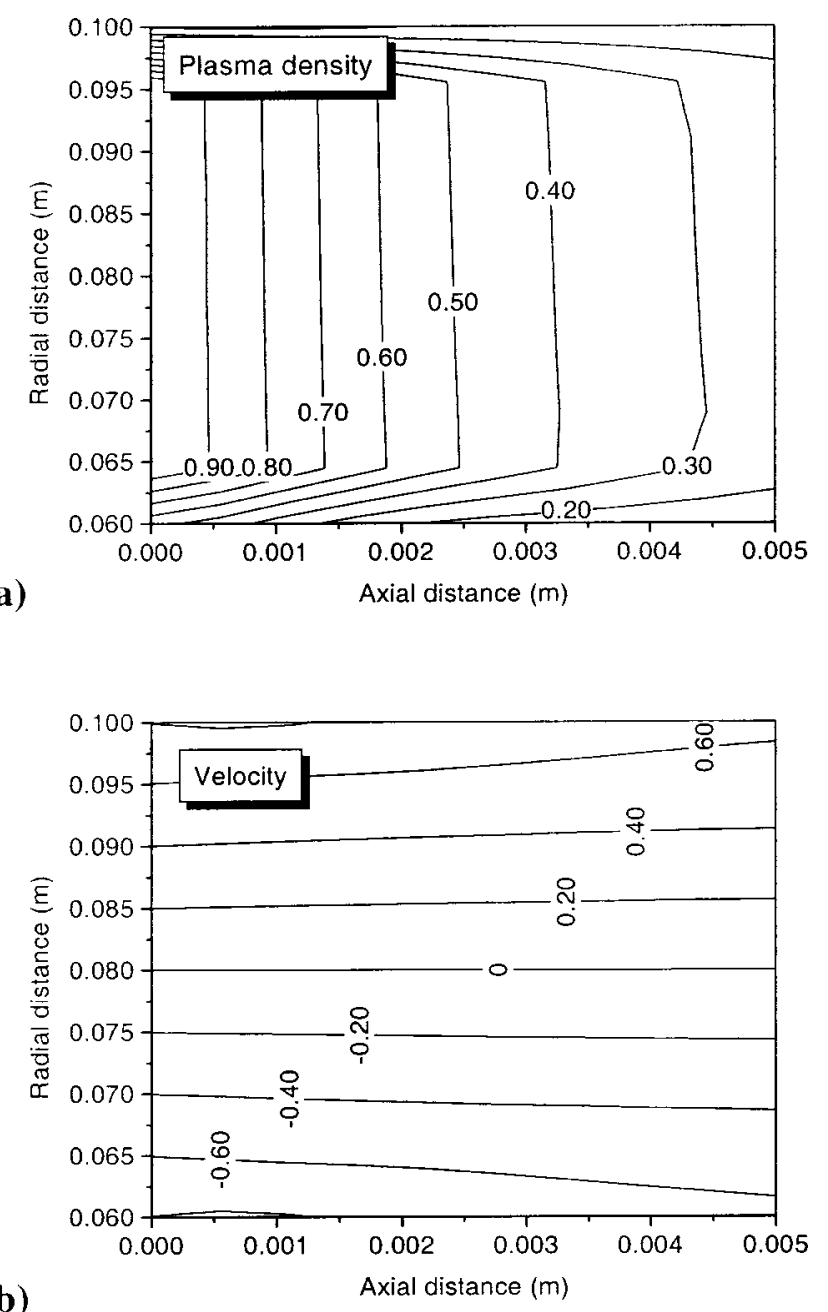

FIG. 2. Plasma parameter distribution in the acceleration channel. (a) plasma density; (b) radial velocity normalized by the Bohm velocity, $\left(T_{e} / m_{i}\right)^{0.5}$

that boundary conditions (see above) rely on assumptions about ionization efficiency in the first stage. In particular, plasma density is used as a parameter. The simulations correspond to the following case: mass flow rate is $20 \mathrm{mg} / \mathrm{s}$, magnetic field is uniform and equals $0.2 \mathrm{~T}$. In this case the calculated discharge current is about $6 \mathrm{~A}$. The particular design is considered that has following geometry: $R_{1}=6 \mathrm{~cm}$, $R_{2}=10 \mathrm{~cm}$, and $L=5 \mathrm{~mm}$. The $2 \mathrm{D}$ hydrodynamic model described in the previous section is used to calculate plasma flow in the second-stage channel.

\section{A. Results}

The computational domain is shown schematically in Fig. 1. Plasma density (normalized by plasma density at the anode) and radial component of ion velocity (normalized by the sound speed) distributions are shown in Fig. 2 in the case of a discharge voltage of $\varphi_{a}=3 \mathrm{kV}$. One can see that a significant density gradient develops in both the radial and axial directions due to ion acceleration. The radial velocity towards the channel walls is about $0.6-0.8$ of the sound speed. 


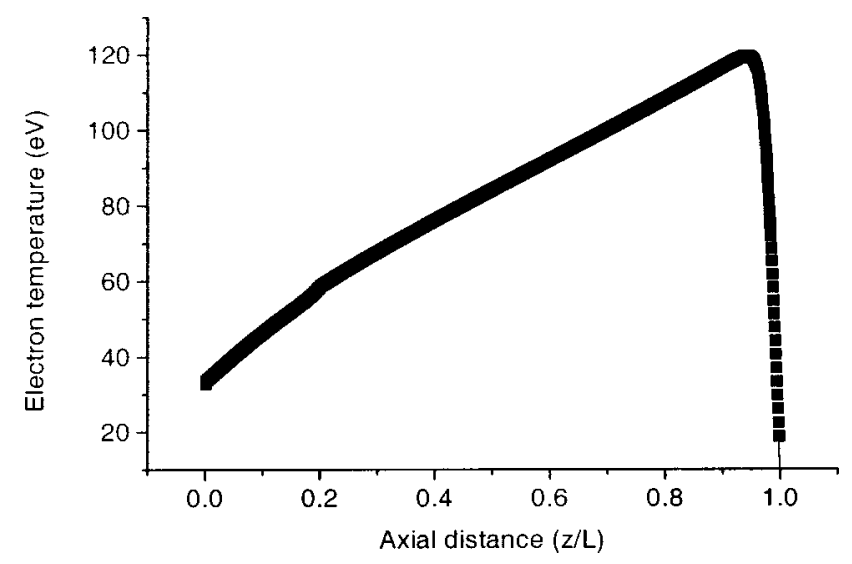

FIG. 3. Electron temperature distribution in the acceleration channel of TAL.

It should be noted that the radial velocity as well as boundary plasma density determine the flux to the wall and therefore the wall erosion rate.

The electron temperature distribution along the channel is shown in Fig. 3 in the case of $\varphi_{a}=3 \mathrm{kV}$. One can see that the electron temperature peaks at about $120 \mathrm{eV}$ near the channel exit plane and then decreases towards the anode. In order to validate the model, some integral characteristics of the thruster are calculated and compared with experiment. The steady-state thrust can be calculated at the thruster exit plane as follows: $T=2 \pi m_{i} \int_{R_{1}}^{R_{2}} n V_{z}^{2} r d r$. The calculated thrust increases linearly with the current (ion beam current) in agreement with experiment, as shown in Fig. 4. It can be seen that the model predicts thrust levels close to those measured experimentally over the entire range of the beam current (or equivalently mass flow rate).

\section{B. Space-charge sheath near the channel wall}

In the channel of the second stage, the guard ring has the cathode potential as shown schematically in Fig. 1. When high voltage across the acceleration channel is considered, one should take into account the importance of the sheath development near the screening walls (guard ring) of the channel. When a negative voltage (cathode potential in the



FIG. 4. Comparison of calculated and measured thrust (experimental data are taken from Ref. 13).

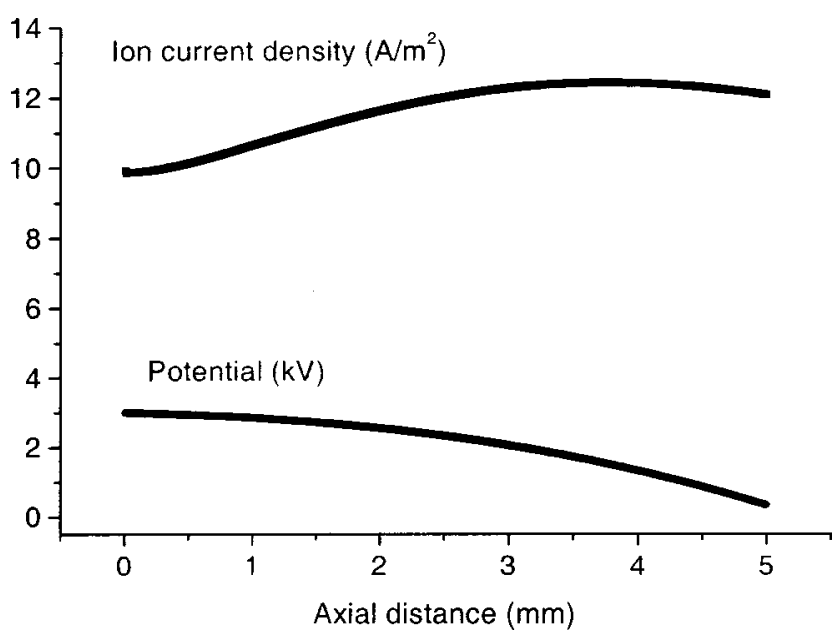

FIG. 5. Ion flux to the wall and plasma potential along the acceleration channel in a TAL. The acceleration voltage is $3 \mathrm{kV}$.

case considered ${ }^{13}$ ) is applied to a surface immersed in a plasma, electrons are repelled from the surface, leading to sheath formation. Electrons drift away from the surface due to the presence of the high electric field. In the steady state, the ions are then accelerated toward the surface by the electric field of the sheath. In the one-dimensional steady-state case the sheath thickness can be estimated according to the Child-Langmuir law ${ }^{26,27}$

$$
s=\left(\frac{4}{9} \epsilon\right)^{1 / 2}\left(\frac{2 e}{m_{i}}\right)^{1 / 4} \frac{U^{3 / 4}}{\left(e Z_{i} N_{s} V_{s}\right)^{1 / 2}},
$$

where $V_{s}$ is the ion velocity at the sheath edge, $U$ is the voltage across the sheath, $s$ is the sheath thickness, $\epsilon$ is the permittivity of vacuum, $N_{s}$ is the plasma density at the sheath edge, and $m_{i}$ is the ion mass. In a TAL, the channel wall has a potential equal to the cathode potential, and therefore the voltage across the sheath, $U$, is equal to the plasma potential and varies along the channel. Similarly, the steadystate sheath thickness in a partially magnetized plasma was calculated in a plasma immersion ion implantation system. ${ }^{28}$ One can see that the steady-state sheath thickness is determined by the plasma density and ion velocity at the sheath edge for a given bias voltage.

Based on the 2D hydrodynamic model, the plasma parameters at the plasma-sheath interface $\left(N_{s}\right.$ and $\left.V_{s}\right)$ as well as ion flux to the wall is calculated. It should be noted that the model used for sheath thickness calculation is one-way coupled, since the 2D plasma flow model does not account for the sheath thickness, while it accounts for the voltage drop in the plasma and the sheath. The ion current density to the wall (radial current) and potential distribution along the channel wall are shown in Fig. 5. One can see that the ion current density to the wall has a maximum near the channel exit. The sheath thickness is several $\mathrm{cm}$ in typical highpower TAL conditions and occupies a significant portion of the channel as shown in Fig. 6. The sheath thickness varies significantly along the channel wall due to variation of the potential drop between the plasma and the wall, which has cathode potential. Note that in typical high-power TAL 


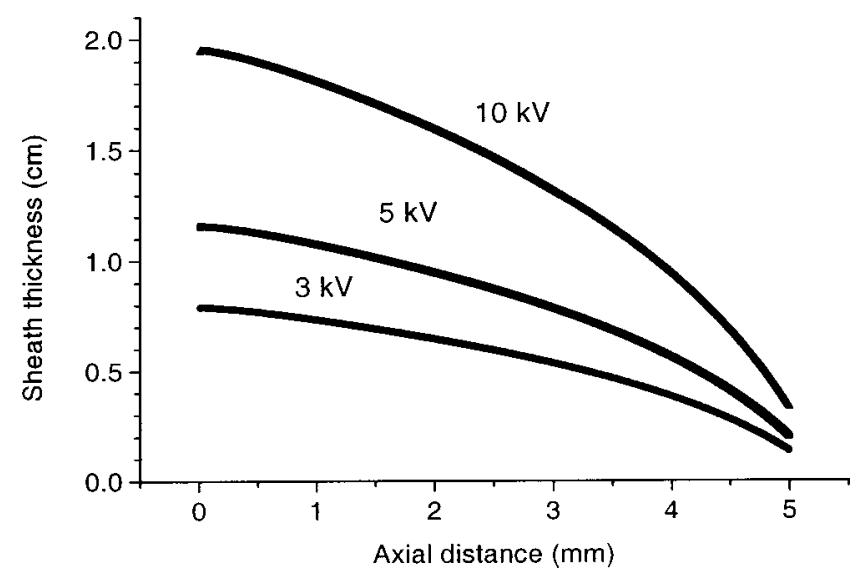

FIG. 6. Sheath thickness as a function of axial position with discharge voltage as a parameter.

$\operatorname{design}^{13}$ the channel width is about $4 \mathrm{~cm}$. It can be seen that when the discharge voltage is about $10 \mathrm{kV}$ almost the entire channel becomes non-quasi-neutral (sheath) and the quasineutral plasma is confined in the middle of the channel. Therefore, one can conclude that the discharge voltage limits the regime of quasineutral acceleration in a TAL with given geometry. Considering the interaction of the highly energetic (due to acceleration in the sheath towards the wall) ion flux with wall materials, one can expect significant erosion.

\section{QUASI-1D MODEL OF THE ANODE LAYER}

It was shown in the previous sections that the effect of the sheath expansion near the acceleration channel wall is very significant in the case of a high-power TAL. It affects mainly the cross-sectional area of the quasineutral plasma as shown in Fig. 6. In turn, the change of the cross-sectional area of the quasineutral plasma affects the current density in the axial direction. It is natural to study this effect using a 1D approach, since the axial current density [Eq. (11)] is calculated in a 1D manner along the channel centerline. To this end, we developed a simplified quasi-1D model of the discharge in crossed $\mathrm{E} \times \mathrm{B}$ fields. The original model of this discharge was developed by Zarinov and Popov. ${ }^{3}$ Recently, a modified version of that model that took into account plasma-wall interactions by introducing a more detailed electron energy equation was developed by Choueiri. ${ }^{7}$ We present here a similar model in which we adopt the formulation of Refs. 3 and 7 except that sheath expansion near the channel wall in the acceleration region is taken into account as it occurs in a high-power TAL. In order to compare with previously published results ${ }^{3,7}$ we adopt here a rectangular coordinate system shown in Fig. 7. The $z$ axis is along the channel axis and the $y$ axis is along the thruster radius, i.e., along the magnetic field. The $x$ axis corresponds to the azimuthal direction in the Hall thruster. In this case, the current density in the quasineutral region will vary along the channel ( $z$ axis). The characteristic time of sheath formation near the channel wall is about the plasma frequency, and therefore a steady-state sheath will be considered. In the case consid-

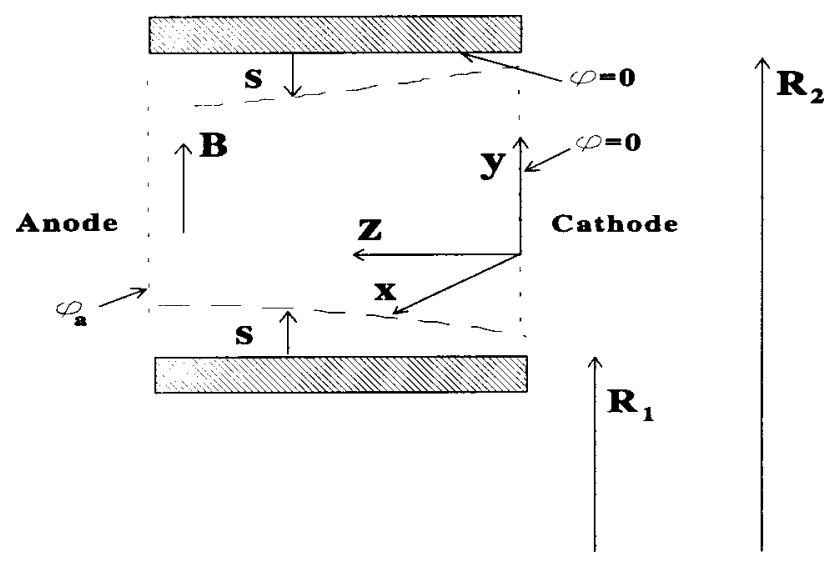

FIG. 7. Schematic of the rectangular coordinate system. The origin of the coordinate system corresponds to the thruster exit plane.

ered, the cross-sectional area $A(z)$ of the quasineutral plasma is varied along the channel. We start with the following set of equations for the quasineutral plasma:

$$
\begin{aligned}
& A \frac{d j_{z}}{d z}+j_{z} \frac{d A}{d z}=e \nu_{i z} n_{e} A, \\
& j_{z}=\mu_{\perp}\left(e n_{e} \frac{d \varphi}{d z}-\frac{d\left(n_{e} T_{e}\right)}{d z}\right), \\
& n_{e}=n_{i}=\frac{j_{i}(z)}{\sqrt{\frac{2 e\left(\varphi_{a}-\varphi\right)}{M}}} \\
& \mu_{\perp}=\frac{e}{m_{e} \nu_{e}} \cdot \frac{1}{1+\left(\omega_{e} / \nu_{e}\right)^{2}},
\end{aligned}
$$

where $\mu_{\perp}$ is the classical cross-field mobility, $j_{i}(z)$ is the ion current dependent on the cross section, $j_{z}$ is the electron current, $\nu_{i z}$ is the ionization frequency, $\nu_{e}$ is the electron collision frequency, and $\omega_{e}$ is the electron cyclotron frequency. The change of the cross-sectional area of the quasineutral plasma due to sheath expansion can be calculated as follows:

$$
A(z)=\pi\left(\left(R_{2}-s\right)^{2}-\left(R_{1}+s\right)^{2}\right),
$$

where $R_{1}$ and $R_{2}$ are the inner and outer radii of the channel (see Figs. 1, 7); $s$ is the sheath thickness (dependent on $z$ ) that can be calculated from Eq. (14). Since the wall has cathode potential (set to 0 for simplicity) the potential drop between the plasma and the wall is simply equal to the plasma potential $\varphi$. The channel cross section at the exit plane $A_{0}$ $=\pi\left(R_{2}^{2}-R_{1}^{2}\right)$ corresponds to the case of zero sheath thickness, since the wall potential is equal to the exit plane potential and therefore the cathode potential.

In addition, following Ref. 3, a simplified energy equation can be used: $T_{e}=\beta \varphi$. It should be noted that similar linear behavior of electron temperature with discharge voltage near the channel exit plane was measured very recently. ${ }^{29}$ On the other hand, the electron temperature profile in the entire channel is much more complicated and is a result of the balance between electron Joule heating and 
cooling due to interaction with the channel walls and ionization. For example, a typical profile is shown in Fig. 3 of this paper. Therefore, linear behavior of the electron temperature with plasma potential is generally only valid near the channel exit. In this particular 1D model we are interested primarily in the effect associated with near-wall sheath expansion. Therefore, in order to simplify the problem we will consider electron temperature as a parameter, which is constant along the channel.

Let us normalize the system of Eqs. (14)-(19). In order to compare results with Refs. 3 and 7 we will perform the same normalizations: $\phi=\varphi / \varphi_{a}, \quad \xi=z / l^{*}, \quad \bar{n}=n_{e} / n^{*}, \bar{j}$ $=j_{e} / j^{*}, \theta=T_{e} / \varphi_{a}$. The characteristic quantities can be defined as follows:

$$
\begin{aligned}
& l^{*}=\sqrt{\frac{e \varphi_{a}}{\frac{\nu_{i z}}{\nu_{e}} m_{e} \omega_{e}^{2}}} ; \quad n^{*}=\frac{j_{i o}}{e \sqrt{\frac{2 e}{M} \varphi_{a}}} ; \\
& j^{*}=e \nu_{i z} n^{*} l^{*} ; \quad \bar{A}=\frac{A(z)}{A_{0}} .
\end{aligned}
$$

The derivative of the channel cross section $A(z)$ can be calculated as follows:

$$
\frac{1}{A} \frac{d A}{d z}=-\frac{2 \frac{d s}{d z}}{\left(\frac{R_{2}^{2}-R_{1}^{2}}{R_{2}+R_{1}}-2 s\right)} .
$$

Finally, the following normalized system of equations is obtained:

$$
\begin{aligned}
& \frac{d \bar{j}}{d \xi}=\bar{n}-\bar{j} \frac{1}{\bar{A}} \cdot \frac{d \bar{A}}{d \xi}, \\
& \bar{j}=\bar{n} \frac{d \phi}{d \xi}-\theta \frac{d \bar{n}}{d \xi}, \\
& \bar{n}=\frac{1}{\bar{A} \sqrt{1-\phi}} .
\end{aligned}
$$

This system of equations is similar to the original formulation of Zharinov and Popov, ${ }^{3}$ with the one exception that change of the cross-sectional area of the quasineutral channel $A(z)$ is taken into account.

Below, the following particular case of a high-power TAL is considered: mass flow rate of $10 \mathrm{mg} / \mathrm{s}$, bismuth as the propellant, geometry of Ref. 13 (i.e., $R_{1}=6 \mathrm{~cm}$ and $R_{2}$ $=10 \mathrm{~cm})$. As was mentioned above, in the calculation presented here we assume a constant electron temperature close to the peak electron temperature $\left(T_{e}=100 \mathrm{eV}\right.$; see Sec. III).

The effect of the near-wall sheath expansion on the anode layer potential profile is shown in Fig. 8. One can see that the sheath expansion strongly affects the potential profile and the anode layer thickness decreases from 0.7 to 0.15 when the sheath expansion effect is taken into account. This happens because the quasineutral plasma region is confined in the middle of the channel and as a result the current den-

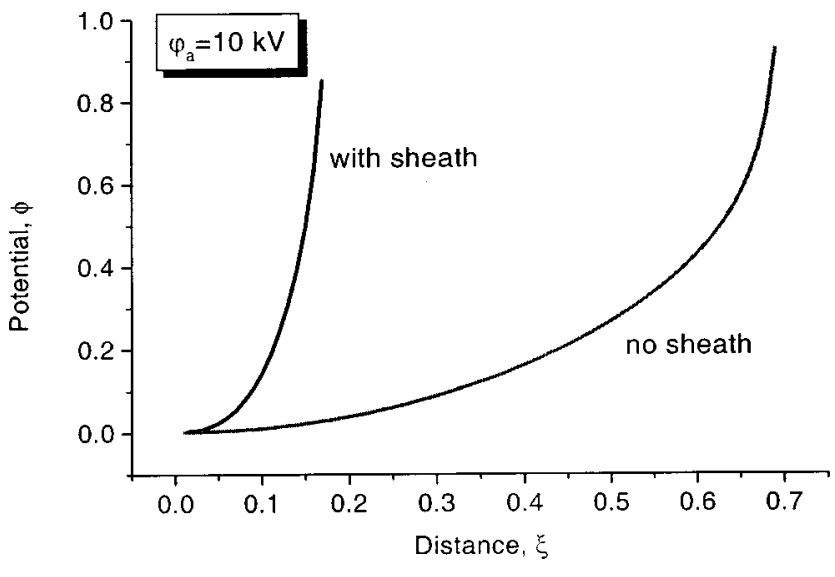

FIG. 8. Plasma potential profile in the quasineutral region with and without sheath expansion. Discharge voltage is $10 \mathrm{kV}$. Origin of the coordinate system $(\xi=0)$ is located at the channel exit plane.

sity increases. In turn, this leads to a higher axial electric field and therefore a smaller anode layer thickness.

The anode layer (acceleration channel) thickness dependence on the discharge voltage is shown in Fig. 9. One can see that the anode layer thickness significantly decreases with the discharge voltage increasing due to the sheath expansion effect. It is interesting to note that shrinking of the anode layer thickness is important, as it leads to a smaller area of contact of the plasma with the walls and therefore smaller total erosion of the channel walls.

It should be noted that the normalized length, $l^{*}$, is proportional to $\sqrt{\varphi_{a}}$, which leads to a nonmonotonic behavior of the anode layer thickness with the discharge voltage in the considered range of discharge voltages. This happens as a result of the near-wall sheath expansion effect. Without considering the near-wall sheath, the dimensional anode layer thickness will monotonically increase with the discharge voltage. Near-wall sheath expansion in the thruster channel leads to the current density increase (see above) and reverses the dependence of the dimensional anode layer thickness on the discharge voltage. The dimensional anode layer thickness will monotonically decrease with the discharge voltage in the large discharge voltage range $(>10 \mathrm{kV})$.

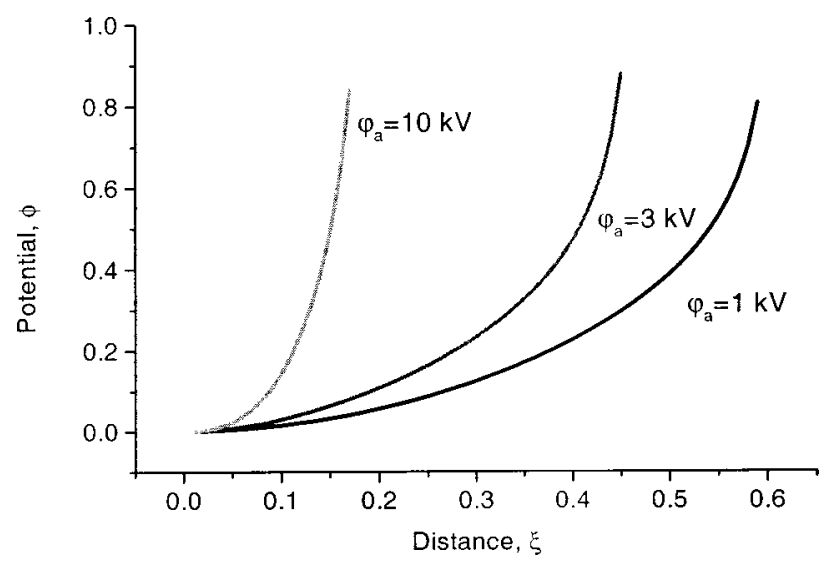

FIG. 9. Plasma potential profile in the quasineutral region with discharge voltage as a parameter. Origin of the coordinate system $(\xi=0)$ is located at the channel exit plane. 


\section{CONCLUDING REMARKS}

In this paper, various aspects of the plasma flow in a high-power thruster with anode layer (TAL) were studied. To this end, a 2D hydrodynamic model of the quasineutral plasma flow and a quasi-1D model of the anode layer were developed. A particular example of a bismuth high-power TAL was considered. One important aspect of plasma-wall interaction in the acceleration channel in TAL is the sheath formation near the channel wall. In a TAL thruster, the channel walls have a potential which is equal to the cathode potential. As a result, a significant potential drop is applied between the wall and the plasma along the magnetic field. This leads to space-charge sheath formation that in typical conditions occupies a significant portion of the channel. It was found that the high-voltage sheath expands significantly, and as a result the quasineutral plasma region is confined in the middle of the channel. For instance, in the case of a 3-10 $\mathrm{kV}$ discharge voltage, the sheath thickness is about $1-2 \mathrm{~cm}$, which is a significant portion of the channel width (which is $4 \mathrm{~cm}$ ). When the quasineutral plasma region is confined in the middle of the channel the current density increases. This leads to higher axial electric field and therefore smaller anode layer thickness. This is an important effect as it leads to a smaller area of contact of the plasma with walls and therefore smaller total erosion. Based on this result, one can suggest a way to control the position of the acceleration region in a high-power Hall thruster by applying voltages to segmented electrodes inside the acceleration channel.

${ }^{1}$ R. J. Etherington and M. G. Haines, Phys. Rev. Lett. 14, 1019 (1965).

${ }^{2}$ G. S. Janes and R. S. Lowder, Phys. Fluids 9, 1115 (1966).

${ }^{3}$ A. V. Zharinov and Yu. S. Popov, Sov. Phys. Tech. Phys. 12, 208 (1967).

${ }^{4}$ A. I. Morozov, Sov. Phys. Dokl. 10, 775 (1966).

${ }^{5}$ V. V. Zhurin, H. R. Kaufman, and R. S. Robinson, Plasma Sources Sci. Technol. 8, 1 (1999).

${ }^{6}$ A. I. Morozov and V. V. Savelyev, in Review of Plasma Physics, edited by B. B. Kadomtsev and V. D. Shafranov (Consultant Bureau, New York, 2000), Vol. 21, p. 203.
${ }^{7}$ E. Choueiri, Phys. Plasmas 8, 5025 (2001).

${ }^{8}$ Y. Raitses, J. Ashkenazy, G. Appelbaum, and M. Guelman, 25th Inter. Conference on Electric Propulsion, Cleveland, $\mathrm{OH}$ (The Electric Rocket Propulsion Society, Worthington, OH, 1997), IEPC 97-056.

${ }^{9}$ Y. Raitses, L. Dorf, A. Litvak, and N. Fisch, J. Appl. Phys. 88, 1263 (2000).

${ }^{10}$ Y. Raitses, D. Staack, A. Smirnov, A. A. Litvak, L. A. Dorf, T. Graves, and N. J. Fisch, 37th AIAA Joint Propulsion Conference, Salt Lake City, UT, 2001 (American Institute of Aeronautics and Astronautics, Washington, DC, 2001), AIAA-2001-3776.

${ }^{11}$ I. I. Beilis, A. Fruchtman, and I. Maron, IEEE Trans. Plasma Sci. 26, 995 (1998).

${ }^{12}$ D. T. Jacobson, R. S. Jankovsky, V. K. Rawlin, and D. H. Manzella, 37th AIAA Joint Propulsion Conference, Salt Lake City, UT, 2001 (American Institute of Aeronautics and Astronautics, Washington, DC, 2001), AIAA2001-3777.

${ }^{13}$ S. Tverdokhlebov, A. Semenkin, and J. Polk, 40th AIAA Aerospace Sciences Meeting, Reno, NV, 2002 (American Institute of Aeronautics and Astronautics, Washington, DC, 2002), AIAA Paper 2002-0348.

${ }^{14}$ M. Keidar and I. D. Boyd, J. Appl. Phys. 86, 4786 (1999).

${ }^{15}$ A. E. Soloduchin and A. V. Semenkin, 28th Inter. Conference on Electric Propulsion, Toulouse, France (The Electric Rocket Propulsion Society, Worthington, OH, 2003), IEPC-2003-0204.

${ }^{16}$ M. Keidar, I. D. Boyd, and I. I. Beilis, Phys. Plasmas 8, 5315 (2001).

${ }^{17}$ I. I. Beilis and M. Keidar, Phys. Plasmas 5, 1545 (1998).

${ }^{18}$ V. S. Erofeev and L. V. Leskov, in Physics and Application of Plasma Accelerators, edited by A. I. Morozov (Minsk, 1974) (in Russian).

${ }^{19}$ A. I. Morozov, Sov. Phys. Dokl. 10, 775 (1966).

${ }^{20}$ J. P. Bouef and L. Garrigues, J. Appl. Phys. 84, 3541 (1998).

${ }^{21}$ N. B. Meezan, W. A. Hargus, and M. A. Cappelli, Phys. Rev. E 63, 026410 (2001).

${ }^{22}$ J. M. Fife and S. Locke, 39th AIAA Aerospace Sciences Meeting, Reno, NV, 2001 (American Institute of Aeronautics and Astronautics, Washington, DC, 2001), AIAA Paper-2001-1137.

${ }^{23}$ E. Ahedo, P. Martinez-Cerezo, and M. Martinez-Sanches, Phys. Plasmas 8, 3058 (2001).

${ }^{24}$ A. I. Morozov and V. V. Savel'ev, Plasma Phys. Rep. 26, 219 (2000).

${ }^{25}$ M. Keidar, I. Beilis, R. L. Boxman, and S. Goldsmith, J. Phys. D 29, 1973 (1996).

${ }^{26}$ C. D. Child, Phys. Rev. 32, 492 (1911).

${ }^{27}$ I. Langmuir, Phys. Rev., Ser. II 2, 450 (1913).

${ }^{28}$ M. Keidar, O. R. Monteiro, A. Anders, and I. D. Boyd, Appl. Phys. Lett. 81, 1183 (2002).

${ }^{29}$ Y. Raitses, D. Staack, L. Dorf, and N. J. Fisch, 39th AIAA Joint Propulsion Conference, Huntsville, AL, 2003 (American Institute of Aeronautics and Astronautics, Washington, DC, 2003), AIAA Paper-2003-5153. 